\title{
Induction of humoral and cellular immune responses against the HIV-1 envelope protein using $\gamma$-retroviral virus-like particles
}

Tea Kirkegaard ${ }^{1}$, Adam Wheatley ${ }^{5}$, Jesper Melchjorsen ${ }^{1}$, Shervin Bahrami ${ }^{2}$, Finn S Pedersen ${ }^{3,4}$, Robert J Center ${ }^{5}$, Damian FJ Purcell ${ }^{5}$, Lars Ostergaard ${ }^{1}$, Mogens Duch ${ }^{3,4}$ and Martin Tolstrup ${ }^{1 *}$

\begin{abstract}
This study evaluates the immunogenicity of the HIV envelope protein (env) in mice presented either attached to $\gamma$ retroviral virus-like-particles (VLPS), associated with cell-derived microsomes or as solubilized recombinant protein (gp160). The magnitude and polyfunctionality of the cellular immune response was enhanced when delivering HIV env in the VLP or microsome form compared to recombinant gp160. Humoral responses measured by antibody titres were comparable across the groups and low levels of antibody neutralization were observed. Lastly, we identified stronger IgG2a class switching in the two particle-delivered antigen vaccinations modalities compared to recombinant gp160.
\end{abstract}

Keywords: HIV-1 envelope protein, Virus-like particles, $\gamma$-retroviruses, immunity

\section{Findings}

The induction of neutralizing antibodies remains key to developing an efficient preventive HIV vaccine. The strongest evidence in support of this comes from nonhuman primate studies, which demonstrate that broadly neutralizing antibodies can protect from infection $[1,2]$. The functional HIV envelope protein (env) complex consists of three heterodimers of the surface subunit gp120 and the transmembrane subunit gp41 arranged in trimeric spikes [3]. Gp120 binds in a sequential manner to $\mathrm{CD} 4$ and to a co-receptor (in most cases either CXCR4 or CCR5) on the target cell. These interactions promote extensive conformational changes in both gp120 and gp41, which leads to fusion of the viral and target-cell membranes. For vaccination purposes antibodies raised to monomeric gp120 antigens primarily target the oligomeric interface, which is not exposed in the functional trimeric structure [4]. Therefore, in order to elicit neutralizing antibodies with high efficacy directed towards the native forms of env, trimeric structure,

\footnotetext{
* Correspondence: martol@rm.dk

'Department of Infectious Diseases, Aarhus University Hospital, Skejby, DK8200 Aarhus N, Denmark

Full list of author information is available at the end of the article
}

ideally in a membrane-bound form should be a crucial property of env vaccine candidates for immunization.

The production and purification of soluble recombinant env proteins having a native trimeric conformation remains challenging [5]. As an alternative, HIV env can be presented as membrane anchored trimers on viruslike particles (VLPs) [6-8]. Several studies imply that membrane-associated trimeric env can raise higher antibody titers with increased neutralization potency compared to soluble recombinant gp120 or gp160 $[9,10]$, and a VLP-based vaccination strategy has previously elicited some strain-specific neutralizing activity in mice and macaques [11].

In order to efficiently pseudotype MLV particles with HIV env, a cytoplasmic tail-truncated HIV env is required $[12,13]$. This limits the retrograde trafficking of HIV env localized on the cell surface by removing endocytosis signals within the cytoplasmic tail of gp41 [14], which in turn enhances env virion incorporation [15]. Importantly, several well-characterized neutralizing antibodies are equally potent in inhibiting both native as well as C-terminal truncated env [16].

The aim of this study was to compare the immunogenicity of env antigens delivered via $\gamma$ - retroviral-like particles consisting of murine leukemia virus (MLV) gag
C Biomed Central

(c) 2011 Kirkegaard et al; licensee BioMed Central Ltd. This is an Open Access article distributed under the terms of the Creative Commons Attribution License (http://creativecommons.org/licenses/by/2.0), which permits unrestricted use, distribution, and reproduction in any medium, provided the original work is properly cited. 
and HIV env (termed HIV env/gag+), a purified cell fraction containing microsomes and HIV env without a viral core (termed HIV env/gag-) and recombinant gp160 (uncleaved env precursor consisting of contiguous gp120 and gp41 domains) produced in H9 cells, detergent solubilized and immuno-affinity purified [17].

We produced Moloney MLV gag particles [18] displaying env (HXB2 strain) with a truncated cytoplasmic tail (termed gp150) as previously described $[13,19]$. Both the VLPs and the microsome-associated HIV env were prepared by transient transfection of $293 \mathrm{~T}$ cells followed by purification via ultracentrifugation of the supernatant through a $20 \%$ sucrose cushion [20]. Western blotting of the sedimented fractions revealed the presence of both precursor gp150 and processed gp120 in HIV env/gag+ as well as HIV env/gag- fractions (Figure 1). Equal amounts of env proteins, as determined by HIV env ELISA, were used for vaccinating mice and compared to rgp160 protein (derived from the HXB2 strain, Autogen-bioclear, UK). Six to eight week-old female BALB/c mice were handled and immunized under SPF conditions at Pipeline Biotech (Trige, Denmark) according to Danish laboratory animal legislation. Three experimental groups of five animals each received two intra-peritoneal injections 4 weeks apart with a final volume of $185 \mu \mathrm{l}$ PBS containing 10 ug of the murine TLR9 ligand CpG oligonucleotide (ODN1826, InvivoGen) as adjuvant. The groups were denoted as either HIV env/gag+ particles, HIV env/gag- microsomes (both receiving $250 \mathrm{ng} /$ injection) or soluble rgp160 (1 ug/injection, Autogen-bioclear, UK). A negative control group of three animals were immunized with PBS alone. Blood samples for preimmune serum production were collected 5 days prior to vaccination. Mice were sacrificed four weeks after the last vaccination and blood and spleens were collected.

IFN- $\gamma /$ IL-2 fluorospot assays (Mabtech, Sweden) were performed on splenocytes purified by ficoll-gradient centrifugation. Splenocytes from all mice were either stimulated with HIV env antigen (rgp160, $50 \mathrm{ng} / \mathrm{mL}$ ) or serum-containing media alone and the number of spot forming units $/ 10^{6}$ splenocytes was determined (Figure 2). Vaccination of mice with HIV env/gag+ (median 143, range 96-439) or HIV env/gag- (median 120, range 60-276) elicited a 10-fold higher env-specific, IFN- $\gamma^{+} \mathrm{T}$ cell response compared with rgp160 vaccinated animals (median 10, range 7-134). For env-specific IFN- $\gamma^{+}$ secreting splenocytes there was a statistically significant increase in the HIV env/gag+ group compared to the rgp160 group $(\mathrm{p}=0.03)$ whereas the increase between the HIV env/gag- vs rgp160 groups failed to reach significance $(p=0.056)$. Similarly, env-specific IL-2 secreting splenocytes were significantly more numerous in both HIV env/gag+ (median 401, range 209-991. p = 0.03 ) and HIV env/gag- (median 517, range 324-820. p

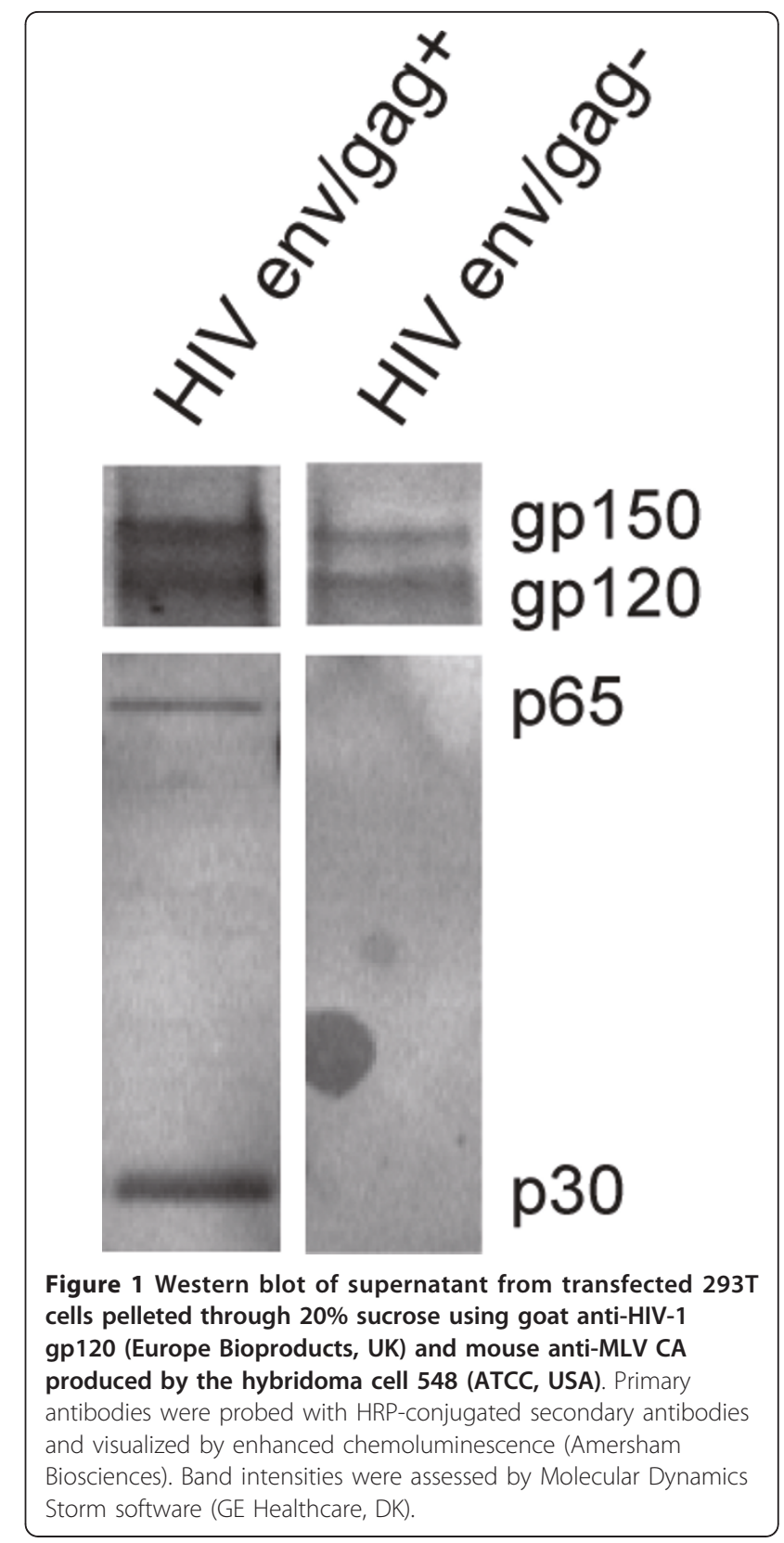

$=0.008)$ vaccinated animals compared with animals receiving rgp160 (median 98, range 267-69) (Figure 2). There were no differences between the two particlesized antigen groups. All statistical group comparisons were performed using a Mann Whitney test.

Analyzing the proportions of env-specific cells secreting both IFN $-\gamma^{+}$and IL-2 $2^{+}$demonstrated higher proportions of dual positive cells in the HIV env/gag+ (5.4\%, p $=0.016)$ and HIV env/gag- $(6.5 \%, \mathrm{p}=0.015)$ groups compared to the rgp160 group (2.12\%, Figure 3$)$. Thus vaccination with membrane-bound HIV env immunogens both with and without a MLV viral core increased both the magnitude and the polyfunctionality of the 


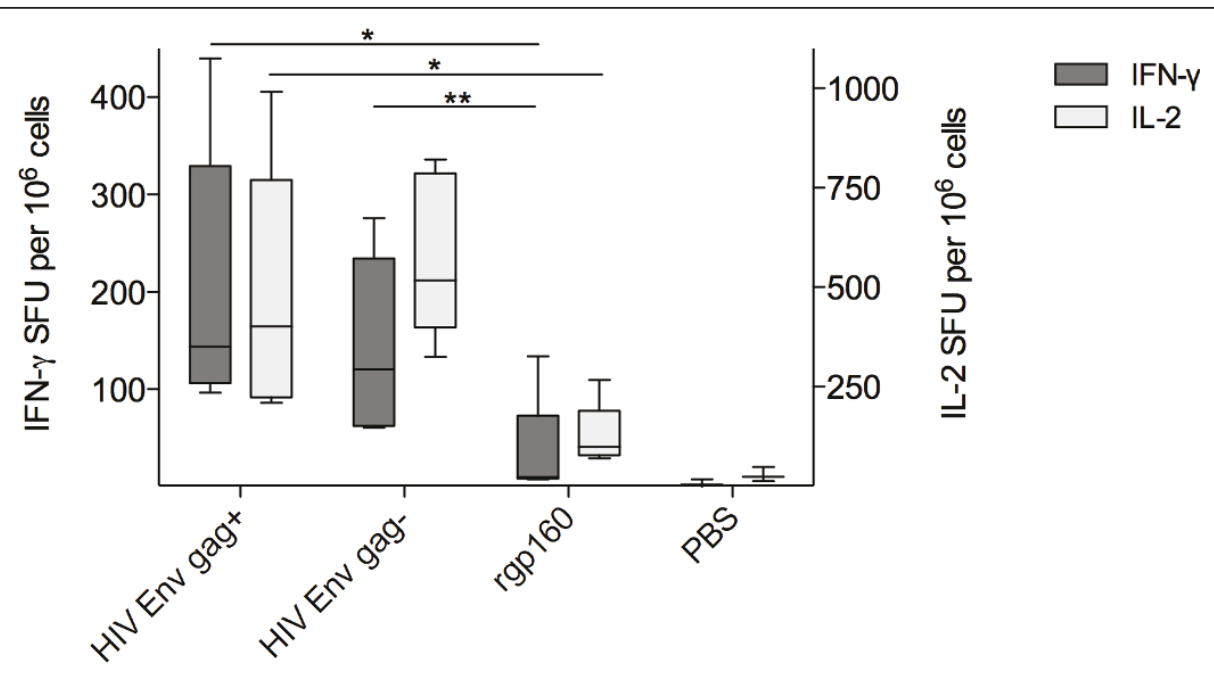

Figure 2 FLUOROspot of splenocytes isolated from BALB/c mice vaccinated twice with VLPs, microsome-associated env (injecting equal amounts as determined by densitometry) or rgp160 (1 ug/injection) four weeks apart. Box and whiskers plot with 5-95\% percentile showing IFN- $\gamma$ (dark) and IL-2 (light) positive counts in the three. Group medians compared with a Mann-Whitney test ${ }^{*} p<0.05 ;{ }^{* *} p<0.01$.

cellular immune response compared to recombinant protein.

The antibody response to the different HIV env immunogens was determined using two different ELISA systems. Firstly, serum IgG from vaccinated mice was analyzed for binding to rgp160. Briefly, Maxisorp

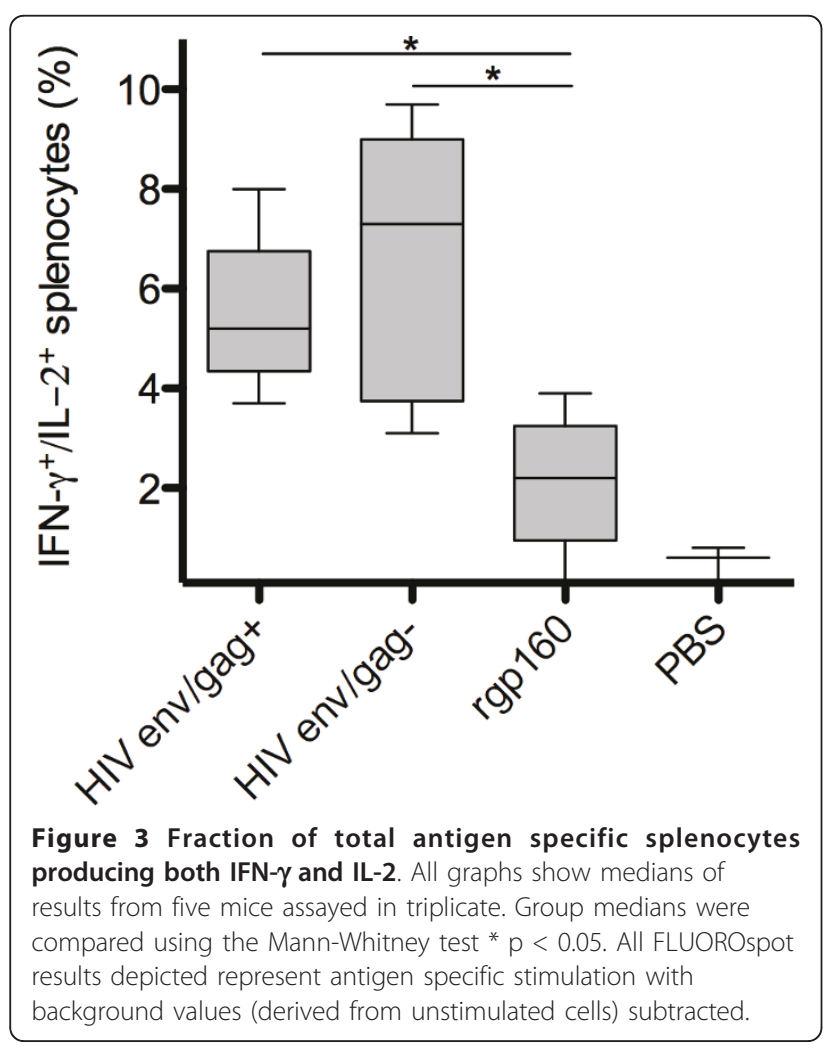

Immunoplates (Nunc, Denmark) were coated with sheep anti-gp120 D7324 antibodies (Aalto Bio Reagents, Ireland) followed by incubation with $44 \mathrm{ng} /$ well rgp160 (Autogen-bioclear, UK) in 5\% low-fat milk PBS 0.1\% Tween. Bound murine antibodies were detected using HRP-conjugated goat anti-mouse IgG (Southern Biotech, USA). The three groups had comparable antigp160 binding titers with mean endpoint dilutions of HIV env/gag + 2500, HIV env/gag- 4500 and rgp160 1400 (Figure 4). A value of at least $2 \times$ the average preimmune sera OD at each dilution from two mice was used as the cut-off for a positive value.

To further address qualitative aspects of the immune response, we determined the HIV env-specific IgG1 to IgG2a isotype ratio in the three experimental groups (Figure 5). Vaccination with either of the particle-sized antigens HIV env/gag+ or HIV env/gag- promoted class-switching of the antibody response to the IgG2a isotype. This is in contrast to vaccination with recombinant gp160 alone. Antibody class-switching to IgG2a has previously been observed in DNA plasmid-based vaccination regimens [21,22]. In the current study, we believe the presentation of membrane-associated env antigens promotes the efficient uptake and cross presentation of env to antigen presenting cells and CD4+ T cells thereby contributing to both the enhanced cellular immunity observed as well as antibody class switching.

Lastly, the ability of sera to neutralize homologous (HXB2) virus infectivity on TZM-bl cells was analyzed. As the challenge virus we utilized HXB2 virus produced in primary PBMC's. Briefly, sera from vaccinated mice and virus were incubated at indicated dilutions for 30 min at 37 C. Subsequently, virus was added to TZM-bl 


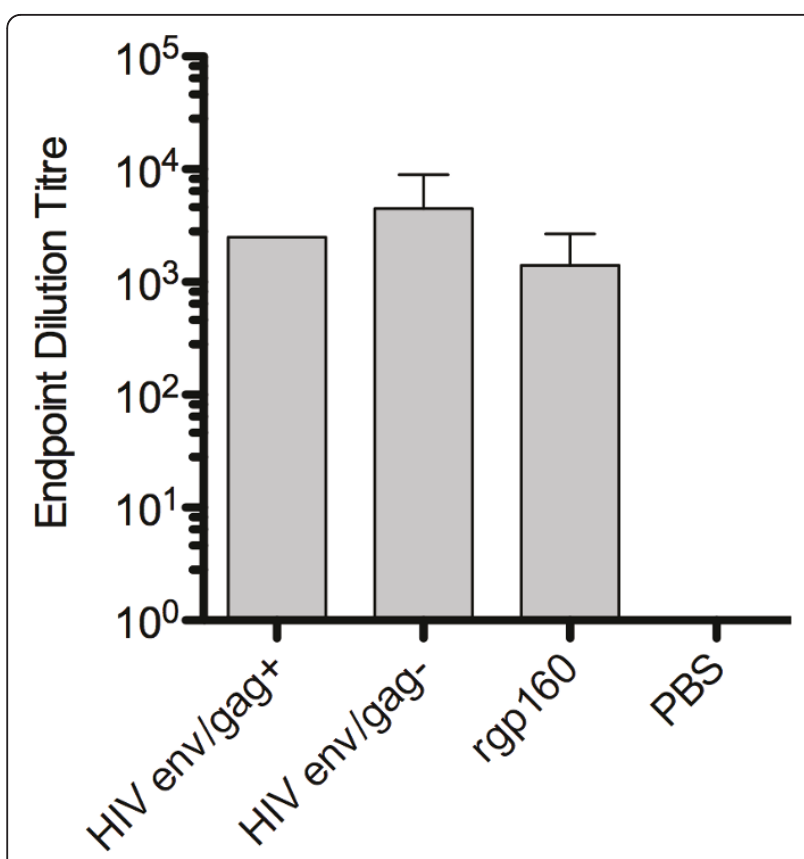

Figure 4 Determination of antibody responses. End-point dilution titre of antibodies binding rgp160. Results are average from five mice $+/$ - SD.

cells and incubated for 48 hrs upon which cells were lysed and luciferase (BriteLite, PerkinElmer, Denmark) activity was determined in a FluorStar Omega (BMG Labtech, Germany). To control for unspecific sera activity pre-vaccination sera were included for each individual mice. Neutralization was calculated as (1-[virus + immune sera/virus + pre-vaccination sera]). Generally, a low degree of neutralizing capability was observed across all three immunization groups (Figure 6). Of interest,

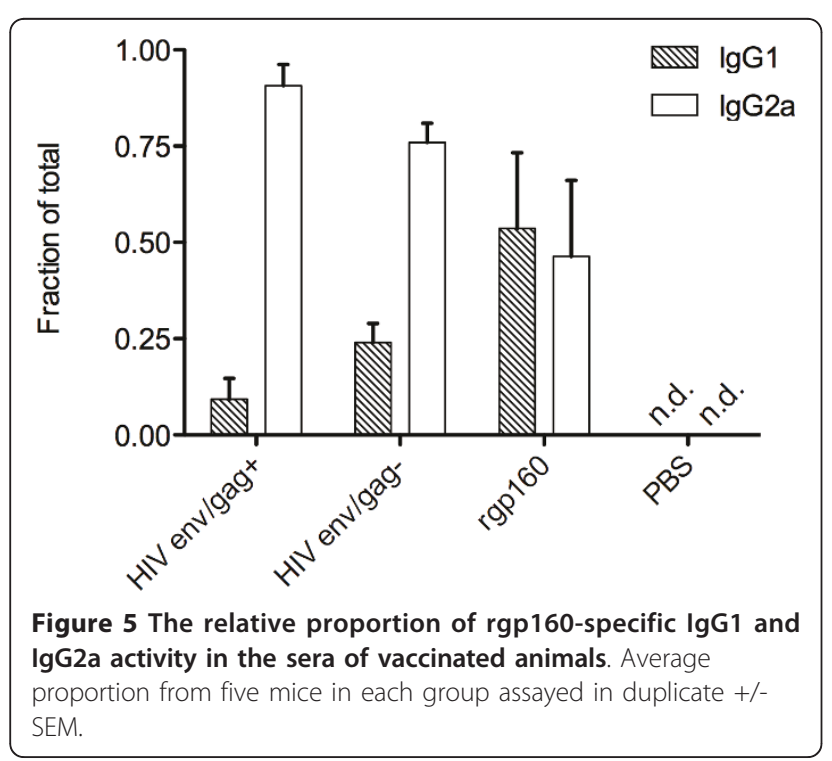

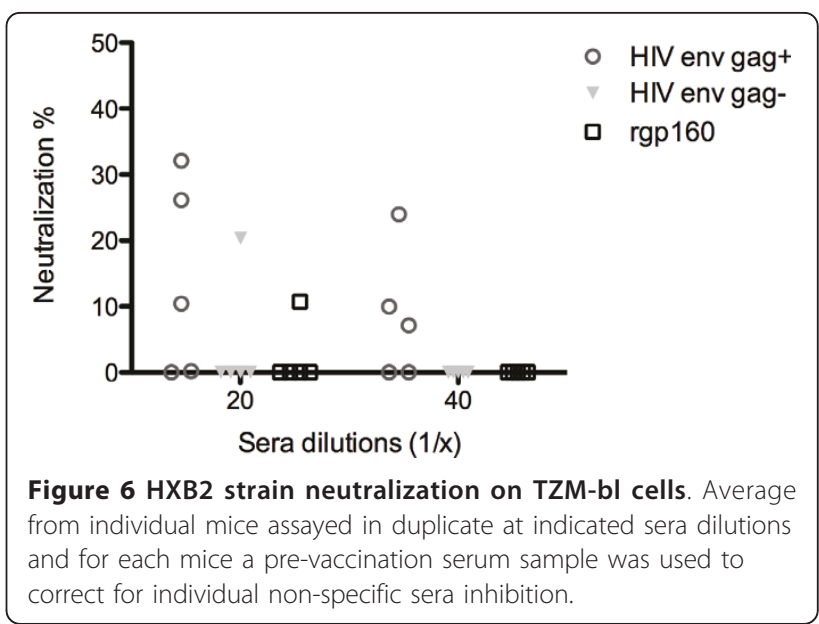

sera from HIV env/gag+ vaccinated animals appeared to display low level of neutralization at higher sera dilutions although no statistical difference was observed.

In conclusion, these results indicate that relative to recombinant protein immunogens, $\gamma$-retroviral-based VLPs and microsome incorporated env can stimulate quantitative and qualitative improvements in $\mathrm{T}$ cell responses targeting HIV env. Thus, a vaccine platform using particle-delivered env trimers appears robust and immunogenic, and also holds advantages as to vector safety. The sequence similarity between $\gamma$-retroviruses and lentiviruses is very small, reducing the potential for recombination. The microsome delivery of antigen appear indistinguishable from the $\gamma$-retroviral-based VLP in terms of immunogenicity but incorporation of env was less efficient posing challenges in terms of larger scale production. Summarized, we believe these immunogenic and safety features support further investigations of particle-delivered HIV env for vaccinations against HIV.

\section{Acknowledgements}

This work was supported by grants the Danish AIDS Foundation, the Scandinavian Society of Antimicrobial Chemotherapy, Region Midtjylland Research Foundation and the Toyota Foundation Denmark. We thank Professor T. Kitamura for the GagPol construct GagPol-IRES-bsr. FluoroSpot plates were kindly enumerated by Staffan Paulie, Mabtech, Sweden.

\section{Author details}

'Department of Infectious Diseases, Aarhus University Hospital, Skejby, DK8200 Aarhus N, Denmark. ${ }^{2}$ SKAU Vaccines, INCUBA Science Park, Brendstrupgaardsvej, DK-8200 Aarhus N, Denmark. ${ }^{3}$ Department of Molecular Biology, University of Aarhus, DK-8000 Aarhus, Denmark. ${ }^{4}$ Interdisciplinary Nanoscience Center, University of Aarhus, DK-8000 Aarhus, Denmark. ${ }^{5}$ Department of Microbiology and Immunology, University of Melbourne, Parkville 3010, VIC, Australia.

\section{Authors' contributions}

TK carried out the preparation of viral-like particles, performed the western blotting, FLUOROspot and antibody ELISA and analyzed the data. AW participated in the western blotting, the FLUOROspot and antibody ELISA. JM participated in the FLUOROspot and antibody ELISA. SB assisted in the 
analysis of data. FSP designed the experiments. RJC designed the experiments and helped to draft the manuscript. DFJP designed the experiments. LO coordinated the study and helped to draft the manuscript. MD conceived the study and participated in the design. MT performed the cloning, assisted in the viral-like particle preparation, designed the experiments analyzed the data and drafted the manuscript. All authors read and approved the final manuscript.

\section{Competing interests}

The authors declare that they have no competing interests.

Received: 12 April 2011 Accepted: 1 August 2011

Published: 1 August 2011

\section{References}

1. Johnson PR, Schnepp BC, Zhang J, et al: Vector-mediated gene transfer engenders long-lived neutralizing activity and protection against SIV infection in monkeys. Nat Med 2009, 15:901-906.

2. Mascola JR, Stiegler G, VanCott TC, et al: Protection of macaques against vaginal transmission of a pathogenic HIV-1/SIV chimeric virus by passive infusion of neutralizing antibodies. Nat Med 2000, 6:207-210.

3. Zhu P, Liu J, Bess J, et al: Distribution and three-dimensional structure of AIDS virus envelope spikes. Nature 2006, 441:847-852.

4. Montefiori DC, Mascola JR: Neutralizing antibodies against HIV-1: can we elicit them with vaccines and how much do we need? Curr Opin HIV AIDS 2009, 4:347-351.

5. Hoxie JA: Toward an antibody-based HIV-1 vaccine. Annu Rev Med 2010, 61:135-152.

6. Crooks ET, Moore PL, Franti M, et al: A comparative immunogenicity study of HIV-1 virus-like particles bearing various forms of envelope proteins, particles bearing no envelope and soluble monomeric gp120. Virology 2007, 366:245-262

7. McBurney SP, Young KR, Ross TM: Membrane embedded HIV-1 envelope on the surface of a virus-like particle elicits broader immune responses than soluble envelopes. Virology 2007, 358:334-346.

8. Quan FS, Sailaja G, Skountzou I, et al: Immunogenicity of virus-like particles containing modified human immunodeficiency virus envelope proteins. Vaccine 2007, 25:3841-3850.

9. Buonaguro L, Visciano ML, Tornesello ML, Tagliamonte M, Biryahwaho B, Buonaguro FM: Induction of systemic and mucosal cross-clade neutralizing antibodies in BALB/c mice immunized with human immunodeficiency virus type 1 clade $A$ virus-like particles administered by different routes of inoculation. J Virol 2005, 79:7059-7067.

10. Hammonds J, Chen X, Fouts T, DeVico A, Montefiori D, Spearman P. Induction of neutralizing antibodies against human immunodeficiency virus type 1 primary isolates by Gag-Env pseudovirion immunization. J Virol 2005, 79:14804-14814.

11. Bellier B, Huret C, Miyalou M, et al: DNA vaccines expressing retrovirus-like particles are efficient immunogens to induce neutralizing antibodies. Vaccine 2009, 27:5772-5780.

12. Mammano F, Salvatori F, Indraccolo S, De Rossi A, Chieco-Bianchi L, Göttlinger HG: Truncation of the human immunodeficiency virus type 1 envelope glycoprotein allows efficient pseudotyping of Moloney murine leukemia virus particles and gene transfer into CD4+ cells. J Virol 1997, 71:3341-3345

13. Schnierle BS, Stitz J, Bosch V, et al: Pseudotyping of murine leukemia virus with the envelope glycoproteins of HIV generates a retroviral vector with specificity of infection for CD4-expressing cells. Proc Natl Acad Sci USA 1997, 94:8640-8645.

14. Byland R, Vance PJ, Hoxie JA, Marsh M: A conserved dileucine motif mediates clathrin and AP-2-dependent endocytosis of the HIV-1 envelope protein. Mol Biol Cell 2007, 18:414-425.

15. Devitt G, Emerson V, Holtkotte D, Pfeiffer T, Pisch T, Bosch V: Incorporation of chimeric HIV-SIV-Env and modified HIV-Env proteins into HIV pseudovirions. Virology 2007, 361:465-471.

16. Kim YB, Lee MK, Han DP, Cho MW: Development of a safe and rapid neutralization assay using murine leukemia virus pseudotyped with HIV type 1 envelope glycoprotein lacking the cytoplasmic domain. AIDS Res Hum Retroviruses 2001, 17:1715-1724.
17. Kalyanaraman VS, Rodriguez V, Veronese F, et al: Characterization of the secreted, native gp120 and gp160 of the human immunodeficiency virus type 1. AIDS Res Hum Retroviruses 1990, 6:371-380.

18. Morita S, Kojima T, Kitamura T: Plat-E: an efficient and stable system for transient packaging of retroviruses. Gene Ther 2000, 7:1063-1066.

19. Tolstrup $M$, Selzer-Plön J, Laursen $A L$, et al: Full fusion competence rescue of the enfuvirtide resistant HIV-1 gp41 genotype (43D) by a prevalent polymorphism (137 K). AIDS 2007, 21:519-521.

20. Cantin R, Diou J, Bélanger D, Tremblay AM, Gilbert C: Discrimination between exosomes and HIV-1: purification of both vesicles from cell-free supernatants. J Immunol Methods 2008, 338:21-30.

21. Center RJ, Wheatley AK, Campbell SM, et al: Induction of HIV-1 subtype B and $A E$-specific neutralizing antibodies in mice and macaques with DNA prime and recombinant gp140 protein boost regimens. Vaccine 2009, 27:6605-6612.

22. Leclerc C, Dériaud E, Rojas M, Whalen RG: The preferential induction of a Th1 immune response by DNA-based immunization is mediated by the immunostimulatory effect of plasmid DNA. Cell Immunol 1997, 179:97-106.

doi:10.1186/1743-422X-8-381

Cite this article as: Kirkegaard et al:: Induction of humoral and cellular immune responses against the HIV- 1 envelope protein using $\gamma$-retroviral virus-like particles. Virology Journal 2011 8:381.

\section{Submit your next manuscript to BioMed Central and take full advantage of:}

- Convenient online submission

- Thorough peer review

- No space constraints or color figure charges

- Immediate publication on acceptance

- Inclusion in PubMed, CAS, Scopus and Google Scholar

- Research which is freely available for redistribution

Submit your manuscript at www.biomedcentral.com/submit
C) Biomed Central 